\title{
Authorship, credit, contribution and problems
}

\author{
Beuy Joob ${ }^{1} \cdot$ Viroj Wiwanitkit ${ }^{2}$
}

Received: 26 November 2015/Revised: 28 November 2015/ Accepted: 28 November 2015/Published online: 7 December 2015

(C) Springer-Verlag Berlin Heidelberg 2015

\section{Dear Editor,}

The recent report of Jia et al. on "authorship, credit and contribution" is very interesting [1]. The authors reported that equally credited authors (ECA) "is increasingly common in major spine journals" [1] and mentioned that "a guideline for authors regarding when and how to designate equal credit is warranted in the future". Indeed, ECA is not the bad thing if the authors actually equally contribute. However, the determination of amount of contribution seems difficult. Warrender recently proposed for the using of scoring system to determine the amount of contribution of each author [2]. Nevertheless, the issue for discussion is not limited to measurement of contribution. The concern should also be on ethical issue. Whether it is an ECA or not, the ethnical problems can be seen in an article with multiple authors [3]. Ghost or gift authorship can be seen and this is common problem in developing countries with rooted culture on seniority [3]. Sometimes, a more complex hidden ethical problem can be seen in article with ECA.
For example, the duplication might be seen and the authors might submit the work for publication in different journals.

\section{Compliance with ethical standards}

Conflict of interest None.

\section{References}

1. Jia Z, Wu Y, Tang Y, Ji W, Li W, Zhao X, Li H, He Q, Ruan D (2015) Equal contributions and credit: an emerging trend in the characterization of authorship in major spine journals during a 10-year period. Eur Spine J (epub ahead of print)

2. Warrender JM (2015) A Simple framework for evaluating authorial contributions for scientific publications. Sci Eng Ethics (epub ahead of print)

3. Teixeira da Silva JA, Dobránszki J (2015) Multiple authorship in scientific manuscripts: ethical challenges, ghost and guest/gift authorship, and the cultural/disciplinary perspective. Sci Eng Ethics (epub ahead of print)
Beuy Joob

beuyjoob@hotmail.com

Sanitation 1 Medical Academic Center, Bangkok, Thailand

2 Hainan Medical University, Haikou, China 